\title{
Quiste aracnoideo de la vaina del nervio óptico simulando defecto glaucomatoso en el campo visual
}

\author{
Optic nerve sheath arachnoid cyst simulating glaucomatous \\ defect in the visual field
}

Edgar Lozano Cruz ${ }^{1}$ ORCID , Ana Milena Bautista Torres ${ }^{2}{ }^{O R C I D}$, Vanessa López Torres $^{3}$ ORCID

Fecha correspondencia:

Recibido: diciembre 26 de 2020.

Revisado: abril 12 de 2021.

Aceptado: abril 16 de 2021.

Forma de citar:

Lozano-Cruz E, Bautista Torres AM, López Torres V. Quiste aracnoideo de la vaina del nervio óptico. Rev CES Med. 2021; 35(1): 44-50.

$\underline{\text { Open access }}$

(C) Derecho de autor

Licencia creative commons

Ética de publicaciones

Revisión por pares

Gestión por Open Journal System

DOl: http://dx.doi.org/10.21615/

cesmedicina.35.1.5

ISSN 0120-8705

e-ISSN 2215-9177
Comparte

คดต

\begin{abstract}
Resumen
Introducción: el quiste aracnoideo es una colección benigna de fluido similar en composición al líquido cefalorraquídeo dentro de la aracnoides, circunscrita por tejido fibrovascular normal que comprime las leptomeninges que rodean el nervio óptico. Se describe el caso de una paciente con quiste aracnoideo de la vaina del nervio óptico con un defecto campimétrico típico de glaucoma, pero con un disco óptico sin características de glaucoma, con el fin de resaltar la necesidad de estudiar con neuroimágenes estos casos y detectar este tipo de alteraciones. El quiste aracnoideo de la vaina del nervio óptico es una entidad excepcional que generalmente tiene un comportamiento benigno, permaneciendo estable en el tiempo, aunque eventualmente puede producir una neuropatía óptica compresiva, afectando la agudeza o el campo visual por daño de la capa de fibras nerviosas. En el caso descrito, este daño se manifestó con un defecto de campo visual que simulaba neuropatía glaucomatosa.
\end{abstract}

Palabras clave: Quistes aracnoideos; Nervio óptico; Escotoma; Glaucoma; Campos visuales.

\begin{abstract}
Background: Arachnoid cyst is a benign fluid collection similar in composition to cerebrospinal fluid within the arachnoid, circumscribed by normal fibrovascular tissue that compresses the leptomeninges surrounding the optic nerve. Objective: To describe the case of a patient with an optic nerve sheath arachnoid cyst with a typical glaucoma campimetric defect, but with an optic disc without findings of glaucoma, to highlight the need to study these cases with neuroimaging to detect this type of changes. Conclusion: Optic nerve sheath arachnoid cyst is an exceptional entity that generally has a benign behavior remaining stable over time; but could eventually originate compressive optic neuropathy, affecting visual acuity or visual fields due to nerve fiber layer damage. In the patient's case this damage was manifested with a visual field defect that simulated glaucomatous neuropathy.
\end{abstract}

Keywords: Arachnoid cysts; Optic nerve; Scotoma; Glaucoma; Visual fields. 


\section{Sobre los autores: \\ 1. Oftalmologo, profesor asistente Universidad del Valle, profesor de pregrado ICESI, especialista en glaucoma del New York Eye and Ear Infirmary. \\ 2. Oftalmóloga especialista en Neuro-oftalmología, Profesora Universidad del Valle. \\ 3. Oftalmóloga Universidad del Valle.}

\section{Introducción}

Un quiste aracnoideo es una colección benigna de fluido similar en composición al líquido cefalorraquídeo (LCR) dentro de la aracnoides (1), circunscrita por una distensión y proliferación de tejido fibrovascular normal que comprime las leptomeninges que rodean el nervio óptico. Histológicamente, se ha observado proliferación de células meningoteliales, una dura engrosada y cuerpos de psammoma (2). Estos quistes suelen encontrarse a lo largo del neuroeje o en la superficie cerebral, con predilección por la fosa craneal media o el ángulo cerebelopontino, sin predilección por sexo (3). Usualmente, no existe ninguna comunicación entre el quiste y el espacio subaraconideo o los ventrículos (4).

Clínicamente, muchos quistes aracnoideos permanecen asintomáticos durante toda la vida, especialmente los más pequeños (5); aunque, dependiendo de su localización, puede presentarse cefalea, vértigos, proptosis, ambliopía, remodelamiento óseo, pérdida de la agudeza visual (6), convulsiones, pérdida de capacidades cognitivas (7), hidrocefalia o anomalías endocrinológicas (8).

Los quistes aracnoideos tienen una prevalencia de hasta 1,1 \% en la población adulta (9), son más comunes en la fosa media y comprenden $0,2 \%$ a $0,9 \%$ de todas las lesiones intracraneales (10); pero el tumor de vainas del nervio óptico más frecuente es el meningioma, típico de mujeres entre 30 y 50 años y supone un $2 \%$ de los tumores orbitarios (11).

La mayoría de los quistes aracnoideos se deben a anomalías del desarrollo que se originan en estadíos tempranos de la etapa embrionaria (6). Los mecanismos que participan en la formación y llenado de los quistes aracnoideos no son bien comprendidos, aunque los datos clínicos, epidemiológicos y de laboratorio indican que los mecanismos genéticos se asocian con su formación (12).

Muchos quistes aracnoideos permanecen asintomáticos, aunque dependiendo de su localización puede presentarse cefalea, vértigos, proptosis, ambliopía, remodelamiento óseo, pérdida de la agudeza visual. convulsiones, pérdida de capacidades cognitivas, hidrocefalia o anomalías endocrinológicas.

Existen tres teorías sobre la formación de los quistes: secreción activa de fluido por las células de la pared del quiste (13), influjo de fluido por gradiente de presión oncótica y, atrapamiento de fluido por mecanismo de válvula. Sin embargo, la teoría más probable podría ser la secreción activa de fluido o transporte activo (14).

Es importante conocer las posibles complicaciones visuales de los quistes aracnoideos de la vaina del nervio óptico, pues pueden producir neuropatía óptica compresiva que podría simular una enfermedad tan importante como el glaucoma. El propósito de este artículo es describir el caso de una paciente con un defecto campimétrico típico de glaucoma en un disco óptico sin características de esta enfermedad, para resaltar sobre la necesidad de estudiar con neuroimágenes estos casos y detectar este tipo de lesiones.

\section{Descripción del caso}

Una mujer de 73 años sin síntomas visuales a quien en cita oftalmológica rutinaria se le encontró disco óptico derecho de aspecto normal y aumento del tamaño de la excavación del disco óptico izquierdo (figuras 1A y 1B), sin hipertensión ocular. La agudeza visual lejana mejor corregida en ojo derecho (OD) fue 20/25 y en ojo izquierdo (OI) 20/20; pupilas simétricas de $4 \mathrm{~mm}$ con reflejos pupilares normales sin defecto pupilar aferente, presión intraocular de ambos ojos (AO) de $13 \mathrm{~mm} / \mathrm{Hg}$. El examen de fondo de ojo reveló excavación del nervio óptico derecho 0,4 x 0,4 e izquierdo 0,4 (horizontal) x 0,6 (vertical). La refracción subjetiva fue: OD: -0,50 -0,75 $x 30^{\circ}$ y Ol: $-0,25-0,75 \times 170^{\circ}$. 
La paciente tenía de diabetes tipo 2, hipertensión arterial y dislipidemia, para lo que recibía metformina, losartán y atorvastatina. Era alérgica a salicilatos, penicilina y analgésicos anti-inflamatorios no esteroideos, y no se reportaban otros antecedentes patológicos.

Se solicitó tomografía óptica de coherencia del nervio óptico, campos visuales computarizados y paquimetría de ambos ojos. La tomografía mostró adelgazamiento del anillo neurorretinal inferior en OD y en el meridiano de la 1 en Ol; el grosor de la capa de fibras nerviosas fue $82 \mu \mathrm{m}$ en OD y $78 \mu \mathrm{m}$ en OI (figura 1C). Los campos visuales fueron realizados con la estrategia Swedish Interactive Threshold Algorithm (por sus siglas en inglés: SITA- Standard 24-2) y fueron confiables en AO: en el OD los falsos positivos fueron $0 \%$, los falsos negativos $2 \%$ y las pérdidas de fijación 0/18; en el 01 los falsos positivos y negativos fueron $0 \%$ y las pérdidas de fijación 1/15.

El campo visual mostró en OD un escotoma arqueado superior (figura 2A) y fue normal en Ol (figura 2B). Los campos visuales descritos en este estudio fueron los primeros que se realizaron y cuatro meses después se realizaron otros, los cuales fueron confiables sin observar cambios respecto a los primeros.

La paquimetría fue en OD $517 \mu \mathrm{m}$ y en Ol $518 \mu \mathrm{m}$.

El examen de fondo de ojo reveló excavación del nervio óptico derecho $0,4 \times 0,4$ e izquierdo 0,4 (horizontal) $\times 0,6$ (vertical). La refracción subjetiva fue: OD: $-0,50-0,75 \times 30^{\circ}$ y Ol: $-0,25-0,75 \times 170^{\circ}$.

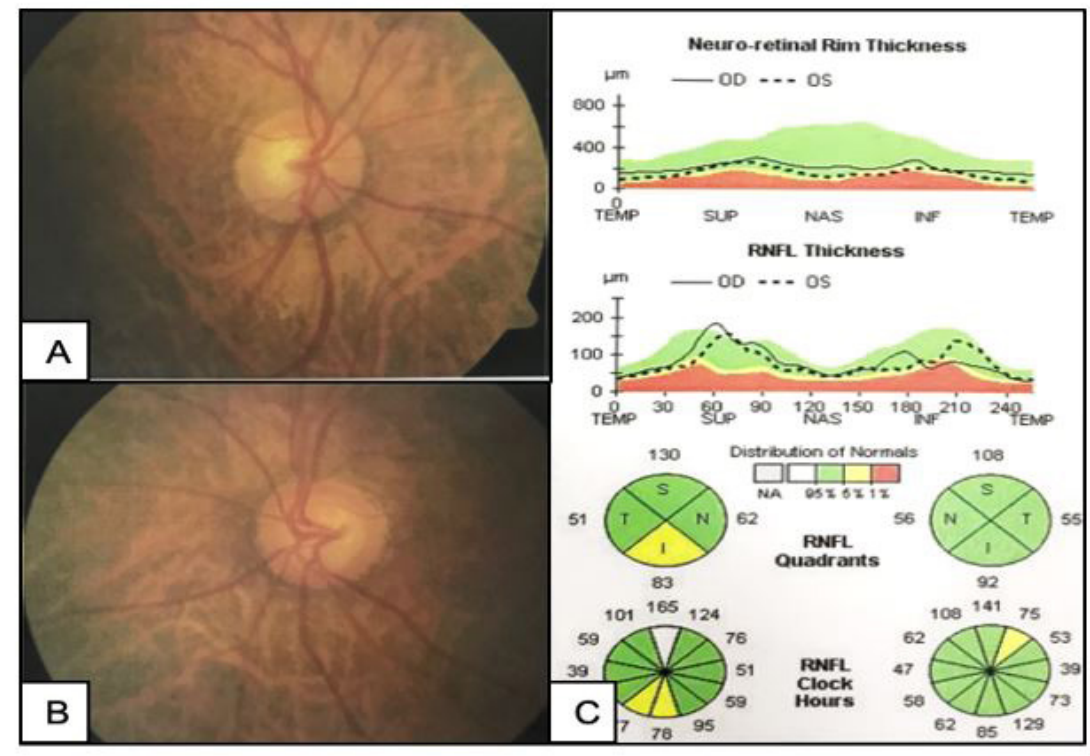

Figura 1. Fotografías de fondo de ojo y tomografía óptica de coherencia del nervio óptico de ambos ojos. A. Fondo de ojo derecho, mostrando excavación de 0,4 × 0,4, fondo miópico. B. Fondo de ojo izquierdo, mostrando excavación de 0,4 (horizontal) y 0,6 (vertical), fondo miópico. C. Tomografía óptica de coherencia del nervio óptico de ambos ojos mostrando adelgazamiento del anillo neurorretinal inferior en el ojo derecho y en el meridiano de la 1 en el ojo izquierdo. Ambos ojos fueron sospechosos de glaucoma en fase inicial, lo cual puede explicar la asimetría de las excavaciones entre ambos ojos. 


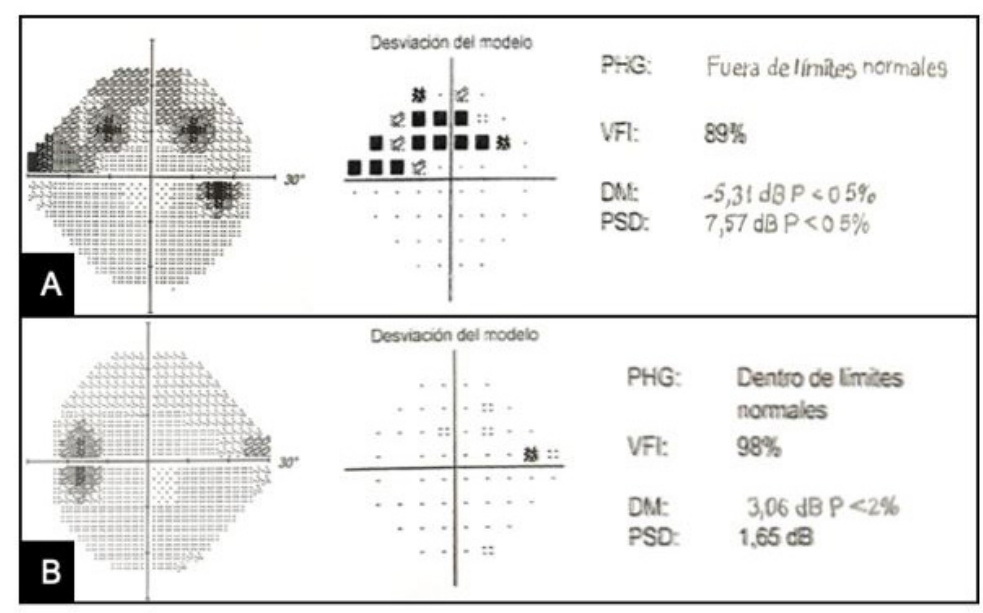

Figura 2. Campos visuales de ambos ojos. A. Campos visuales del ojo derecho mostrando un escotoma arqueado superior. B. Campos visuales del ojo izquierdo normales.

La resonancia magnética mostró una formación quística con señal similar al LCR, a nivel de la vaina del nervio óptico derecho de aproximadamente 3,4 mm de diámetro transverso por 10 mm de diámetro longitudinal. El quiste producía compresión y desplazamiento hacia arriba del nervio óptico, altamente sugestivo de quiste aracnoideo de la vaina del nervio óptico.
Debido a que no había aumento de la excavación del nervio óptico derecho, a que la tomografía óptica de coherencia del OD mostró adelgazamiento de la capa de fibras nerviosas inferiores y a que el campo visual se correlacionó con este defecto que permaneció sin cambios en el tiempo, mientras que el campo visual izquierdo no mostró defectos, se descartaron causas compresivas del nervio óptico, para lo cual se solicitó una resonancia magnética con gadolinio de órbitas tomada después de un periodo de seguimiento de cinco meses, que fue realizada en un equipo de 3 Tesla para descartar lesiones vasculares que pudiesen comprometer el nervio óptico.

La resonancia magnética mostró una formación quística con señal similar al LCR, a nivel de la vaina del nervio óptico derecho de aproximadamente $3,4 \mathrm{~mm}$ de diámetro transverso por $10 \mathrm{~mm}$ de diámetro longitudinal. El quiste producía compresión y desplazamiento hacia arriba del nervio óptico, altamente sugestivo de quiste aracnoideo de la vaina del nervio óptico (figuras 3 A-D).
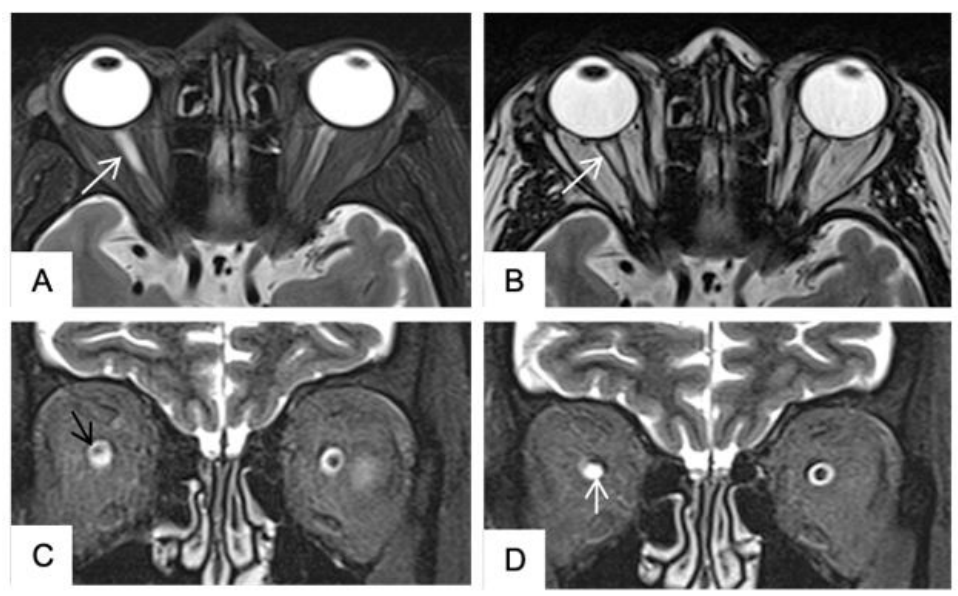

Figura 3. Resonancia magnética de órbitas. A y B. Cortes transversales en T2 sin supresión grasa (A) y con supresión grasa (B), que muestran la presencia de lesión quística (flecha blanca) con señal similar al LCR en la vaina del nervio óptico derecho. C y D. Cortes coronales en T2 con supresión grasa. Muestra la lesión quística en la vaina del nervio óptico derecho (flecha negra) (C), que comprime y desplaza superiormente el nervio óptico, compatible con quiste aracnoideo (flecha blanca) (D). 
Enero - abril de 2021 - Pág 48

\section{Discusión}

La alteración de los campos visuales simulando un defecto glaucomatoso pero con un nervio óptico sin características de glaucoma debe sugerir una enfermedad diferente de esta última, por lo cual es importante buscar otras causas mediante estudios adicionales. En el presente caso, el nervio óptico derecho era de aspecto normal aunque el campo visual sí sugería glaucoma; esta poca correspondencia condujo a descartar compresión del nervio óptico, mediante estudio imagenológico. En la resonancia magnética de órbitas se observó quiste aracnoideo de la vaina del nervio óptico derecho, vinculando la ubicación de la compresión con la alteración en el campo visual ipsilateral.

Hallazgos similares se han observado en otros casos reportados donde la compresión de las fibras inferiores del nervio óptico generaron un escotoma en cuadrantes superiores del ojo afectado, con disminución de la agudeza visual (15) y defectos arqueados que simulaban glaucoma (16). Las neuropatías ópticas adquiridas pueden resultar en cualquier clase de defecto del campo visual, siendo mas comunes los escotomas centrales, cecocentrales, arqueados o altitudinales (17).

La pérdida de agudeza visual secundaria a un quiste aracnoideo puede estar causada por dos mecanismos diferentes: presión directa sobre el nervio óptico con o sin

La pérdida de agudeza visual secundaria a un quiste aracnoideo puede estar causada por presión directa sobre el nervio óptico o por incremento de la presión intracraneana causada por el quiste. tracción de estructuras nerviosas a lo largo de la vía óptica desde el nervio óptico hasta la corteza occipital o, indirectamente, por incremento de la presión intracraneana causada por el quiste. El primer mecanismo puede causar disminución de la agudeza visual en un solo ojo, hemianopsia bitemporal si lesiona el quiasma óptico o hemianopsia homónima si esta detrás de este. Por otro lado, el aumento de la presión intracraneana puede causar disminución de la agudeza visual debido al edema papilar que eventualmente llevará a una atrofia óptica y ceguera (4).

Una vez se tenga la sospecha clínica de lesión compresiva del nervio óptico deben realizarse estudios de imagen de alta resolución, con los que un quiste aracnoideo puede visualizarse como una imagen lobulada o quística, pero con nervio óptico indemne y sin calcificaciones intralesionales (18).

Se prefiere la resonancia magnética como elemento esencial para validar el diagnóstico (19), en la que se utilizan secuencias de supresión grasa aumentando el valor diagnóstico de lesiones de tejidos intraorbitarios o compresión del nervio óptico (20). Debido a que el quiste aracnoideo de la vaina del nervio óptico es una entidad rara, el diagnóstico se realiza una vez se han descartado otras causas más frecuentes, como meningioma o glioma del nervio óptico (21).

La paciente no presentaba alteración de la visión central, pues el compromiso era de fibras arqueadas y los quistes aracnoideos típicamente mantienen un tamaño estable en el tiempo. Eventualmente, podría presentarse pérdida visual central irreversible aguda o progresiva por daño de las fibras nerviosas del haz papilomacular, por lo que es necesario el seguimiento oftalmológico. Los síntomas de efectos de masa son raros y la expansión sintomática del quiste puede relacionarse, entre otras causas, con hemorragia intraquística (22). 


\section{Conclusiones}

Es recomendable estudiar con imágenes a los pacientes que presenten signos 0 síntomas de riesgo de lesiones compresivas, como defectos del campo visual que no correspondan con el aspecto del disco óptico, pérdida de la agudeza visual en un solo ojo, hemianopsias, discos excavados con palidez del anillo neurorretinal o alteraciones neurológicas asociadas a defectos campimétricos. Cuando la sospecha clínica sea muy alta, la resonancia magnética de órbitas o angiorresonancia de órbitas son estudios útiles para detectar lesiones que compriman el nervio óptico.

\section{Aspectos éticos}

Se trata de un reporte de caso descriptivo retrospectivo, sin riesgo para la paciente. Para ello, se obtuvo consentimiento informado para la publicación del caso. Los autores no declaran conflictos de interés y no hubo financiación por parte de ninguna entidad.

\section{Bibliografía}

1. Sung MS, Park SW, Heo H. Arachnoid cyst accompanied by proptosis and unilateral high myopia. Int Ophthalmol. 2014;34(3):689-92.

Es recomendable estudiar con imágenes a los pacientes que presenten signos o síntomas de riesgo de lesiones compresivas, La resonancia magnética de órbitas y la angiorresonancia de órbitas son estudios útiles para detectar lesiones que compriman el nervio óptico.
2. Akor C, Wojno TH, Newman NJ, Grossniklaus HE. Arachnoid cyst of the optic nerve: report of two cases and review of the literature. Ophthal Plast Reconstr Surg. 2003;19(6):466-9.

3. Bonneville JF, Bonneville F, Cattin F, Nagi S. Preface. In: MRI of the pituitary gland. Springer International Publishing Switzerland; 2016. p. vii.

4. Menon RK. Arachnoid Cyst and Visual Function. In: Arachnoid cysts: clinical and surgical management. Elsevier Inc.; 2018. p. 29-43.

5. Temblador-Barba I, Gálvez-Prieto-moreno C, Martínez-Jiménez M. Arachnoid cyst of the optic nerve: Therapeutic management and progress. Asian J Ophthalmol. 2020;17(2):137-41.

6. Fisher T, Nugent R, Rootman J. Arachnoid cysts with orbital bone remodeling Two interesting cases. Orbit. 2005;24(1):59-62.

7. Wester K. Intracranial arachnoid cysts - do they impair mental functions? J Neurol. 2008;255(8):1113-20.

8. Schroeder H. Arachnoid cysts. In: Sindou M, editor. Practical Handbook of Neurosurgery. Vienna: Springer; 2009.

9. Vernooij M, Ikram A, Tanghe H, Vincent A, Hofman A, Krestin GP, et al. Incidental findings on brain MRI in the general population. N Engl J Med. 2007;357(18):1821-8.

10. Pradilla G, Jallo G. Arachnoid cysts: Case series and review of the literature. Neurosurg Focus. 2007;22(February):1-4.

11. Eddleman CS, Liu JK. Optic nerve sheath meningioma: current diagnosis and treatment. Neurosurg Focus. 2007;23(5):1-7. 
12. Aarhus M, Helland CA, Lund-Johansen M, Wester K, Knappskog PM. Microarray-based gene expression profiling and DNA copy number variation analysis of temporal fossa arachnoid cysts. Cerebrospinal Fluid Res. 2010;7:2-9.

13. Helland CA, Aarhus M, Knappskog P, Olsson LK, Lund-Johansen Morten M, Amiry-Moghaddam M, et al. Increased NKCC1 expression in arachnoid cysts supports secretory basis for cyst formation. Exp Neurol. 2010;224(2):424-8.

14. Berle M, Wester KG, Ulvik RJ, Kroksveen AC, Haaland ØA, Amiry-Moghaddam M, et al. Arachnoid cysts do not contain cerebrospinal fluid: A comparative chemical analysis of arachnoid cyst fluid and cerebrospinal fluid in adults. Cerebrospinal Fluid Res. 2010;7:1-5.

15. Kural C, Kullmann M, Weichselbaum A, Schuhmann MU. Congenital left temporal large arachnoid cyst causing intraorbital optic nerve damage in the second decade of life. Child's Nerv Syst. 2016;32(3):575-8.

16. Wegener M, Prause JU, Thygesen J, Milea D. Arachnoid cyst causing an optic neuropathy in neurofibromatosis 1: Diagnosis/Therapy in Ophthalmology. Acta Ophthalmol. 2010;88(4):497-9.

17. Atkins EJ, Newman NJ, Biousse V. Lesions of the optic nerve. Handbook of Clinical Neurology. Elsevier B.V.; 2011. 159-184 p.

18. Genol I, Troyano J, Ariño M, Iglesias I, Arriola P, García-Sánchez J. Meningocele, glioma y meningioma del nervio óptico: Diagnóstico diferencial y tratamiento. Arch Soc Esp Oftalmol. 2009;84(11):563-8.

19. Weber AL, Caruso P, Sabates NR. The optic nerve: Radiologic, clinical, and pathologic evaluation. Neuroimaging Clin N Am. 2005;15(1):175-201.

20. Halimi E, Wavreille O, Rosenberg R, Bouacha I, Lejeune JP, Defoort-Dhellemmes S. Optic nerve sheath meningocele: A case report. Neuro-Ophthalmology. 2013;37(2):78-81.

21. Mesa JC, Muñoz S, Arruga J. Optic nerve sheath meningocele. Clin Ophthalmol. 2008;2(3):661-4.

22. Cincu R, Agrawal A, Eiras J. Intracranial arachnoid cysts: Current concepts and treatment alternatives. Clin Neurol Neurosurg. 2007;109(10):837-43. 